\title{
Intracranial pressure estimation by palpation of the anterior fontanelle
}

\author{
A M KAISER AND A G L WHITELAW \\ Department of Paediatrics and Neonatal Medicine, Royal Postgraduate Medical School, Hammersmith \\ Hospital, London
}

SUMMARY Simultaneous assessment of intracranial pressure by anterior fontanelle palpation and direct measurement from a cerebrospinal fluid cannula showed that mean pressure was significantly different between 'soft' (5.4 mm Hg) and 'tense' (14.0 mm Hg) fontanelles, but with considerable overlap. Normal fontanelle tone was often confirmed, whereas clinical assessment of raised intracranial pressure was unreliable.

Measurement of intracranial pressure is of great importance in the neonatal period because of the number of conditions in which it may be raised, the possible consequences of its rise, and the efficacy of modern methods of treatment. Conventionally, intracranial pressure is assessed by palpating the anterior fontanelle with the finger. ${ }^{1} 2$ The rationale is that the bony defect of the anterior fontanelle acts as a pressure vent, displaced centrifugally by an increase in intracranial pressure.

In comparison with invasive measurements of intracranial pressure in the newborn, fontanelle palpation has the advantages of being simple to perform, cheap, and free from adverse effects. Its only disadvantage is the inevitably intermittent nature of the assessment. Despite claims of its reliability, ${ }^{3}$ there are no published reports comparing actual intracranial pressure and results obtained by clinical assessment.

\section{Patients and methods}

During the study of intracranial pressure in normal and abnormal neonates, 17 babies in our neonatal unit underwent invasive measurement of intracranial pressure at the time of clinically indicated removal of cerebrospinal fluid (CSF). Their gestational ages ranged from 24 to 41 weeks. The intracranial pressure was measured precisely by a Gaeltec pressure transducer (Gaeltec Ltd, Dunvegan, Isle of Skye, Scotland) coupled to a Tektronix monitor (Tektronix Inc, PO Box 500, Beaverton, Oregon 97077, United States), which was connected by a short length of non-compliant tubing to a CSF cannula (a 23 gauge butterfly or $22 \mathrm{G}$ spinal needle) placed in the lumbar subarachnoid space or lateral cerebral ventricles as clinically indicated. ${ }^{4}$ Care was taken to establish the presence of craniospinal communication for lumbar pressures. ${ }^{4}$ Pressure was recorded when the baby was at rest in a lateral horizontal posture and referred to the level of the right atrium.

The anterior fontanelles of the babies were palpated by one of us (AMK) before the precise measurement on 59 occasions, while the baby was horizontal and at rest. At the time of measurement, the babies' postmenstrual ages varied from 28 to 80 weeks and chronological ages from 1 day to 53 weeks. All fontanelles were at least double the area of the fingertip, so the difficulties commonly encountered in assessment of small fontanelles did not occur. The fontanelle tension was subjectively classified as 'soft', 'full', or 'tense', according to the resistance to gentle digital pressure.

The distribution of precise measurements of pressure between each fontanelle tension group was compared by Student's unrelated $t$ test.

\section{Results}

The fontanelle tension was classified as soft on 31 occasions, full on 14, and tense on 14 .

The distributions of invasive measurements were skew to the right, so they were converted to Gaussian by taking logarithms to base 10 (Figure). Analysis was performed after this transformation, so the range is described as mean ( 2 standard deviations) to overcome the difficulty of describing log results. The means (ranges) were $5.4(1.9-15.7) \mathrm{mm} \mathrm{Hg}$ for the soft group, $8.8(2 \cdot 6-30 \cdot 2) \mathrm{mm} \mathrm{Hg}$ for the full group, and 14.0 (3.3-59.3) $\mathrm{mm} \mathrm{Hg}$ for the tense group. The means differed at the $0.1 \%$ level for the soft and tense groups and at the $2 \%$ level for the soft and full groups; the full and tense groups were not significantly different.

There was considerable overlap between the groups. For example, $9.7 \%$ of all the pressures recorded in the soft group were $>10 \mathrm{~mm} \mathrm{Hg}$, commonly taken to be the level of pressure at which intervention is indicated. ${ }^{5}$ Conversely, $50 \%$ of all those in the tense group were $<10 \mathrm{~mm} \mathrm{Hg}$. 


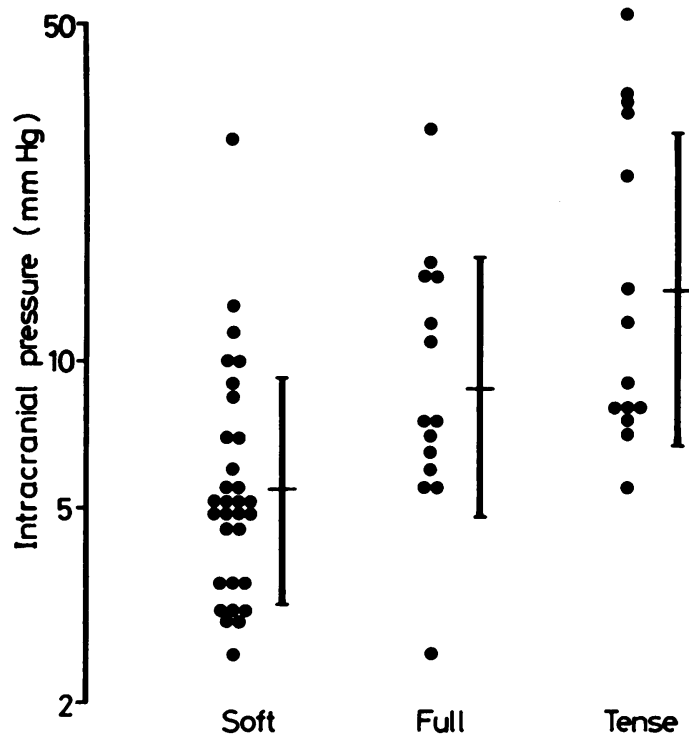

Fontanelle tension group

Figure Intracranial pressure (logarithmic scale) in babies with soft, full, or tense anterior fontanelles. The horizontal bar represents the mean and the vertical bar one standard deviation.

\section{Discussion}

We have shown that there is some agreement between the precise intracranial pressure value and its assessment by palpation, in so far as there was a significantly different distribution of pressures between the soft and tense and between the soft and full fontanelle tension groups. The clinical usefulness is limited, however, by the broad overlap between the distributions, even if the intermediate full group is ignored. This overlap would have led to the incorrect management of $9.7 \%$ of the soft fontanelle group and $50 \%$ of the tense fontanelle group, which altogether comprised $22 \%$ of the sample in those two groups.

No greater accuracy of digital assessment was found even when one baby was assessed over the course of time. Thus the variability was not only limited to the group as a whole but also to individual babies. This was not related to the gestational, chronological, or postmenstrual ages of the subjects.

The most likely reasons for the unreliability in palpation of fontanelle tension are the variability of skin hydration and of the compliance of the scalp and dura between babies and at different times in the same baby.

These findings suggest that it is not safe to rely on the digital assessment of fontanelle tension when treatment of raised intracranial pressure is under consideration. On the other hand, in the group considered clinically unequivocally normal, about $90 \%$ of the invasive measurements were at an acceptable level.

Mr J Messeguer maintained the electronic equipment. The nurses on the neonatal unit helped in the performance of pressure measurements. AMK was supported by Birthright.

\section{References}

'Bell WE, McCormick WF. Increased intracranial pressure in children. In: Schaffer AJ, ed. Major problems in clinical pediatrics. Vol VIII. 2nd ed. Philadelphia: W B Saunders, 1978:4.

2 Forrest DM, Tsingoglou S. The false fontanelle as a practical method of long-term testing of intracranial pressure. Dev Med Child Neurol [Suppl] 1968;16:17-20.

3 Welch $K$. The intracranial pressure in infants. $J$ Neurosurg 1980;52:693-9.

4 Kaiser AM, Whitelaw AGL. Normal cerebrospinal fluid pressure in the newborn. Neuropediatrics 1986;17:100-2.

5 Levene MI, Evans DH. Medical management of raised intracranial pressure after severe birth asphyxia. Arch Dis Child 1985;60:12-6.

Correspondence to $\operatorname{Dr}$ A $M$ Kaiser, The Hospital for Sick Children, Great Ormond Street, London WC1.

Received 17 November 1986 